\title{
DEGREES OF SUMS IN A SEPARABLE FIELD EXTENSION
}

\section{M. ISAACS}

Let $F$ be any field and suppose that $E$ is a separable algebraic extension of $F$. For elements $\alpha \in E$, we let $\operatorname{dg} \alpha$ denote the degree of the minimal polynomial of $\alpha$ over $F$. Let $\alpha, \beta \in E, \operatorname{dg} \alpha=m, \operatorname{dg} \beta=n$ and suppose $(m, n)=1$. It is easy to see that $[F(\alpha, \beta): F]=m n$, and by a standard theorem of field theory (for instance see Theorem 40 on p. 49 of [1]), there exists an element $\gamma \in E$ such that $F(\alpha, \beta)$ $=F(\gamma)$ and thus $\operatorname{dg} \gamma=m n$. In fact, the usual proof of this theorem produces (for infinite $F$ ) an element of the form $\gamma=\alpha+\lambda \beta$, with $\lambda \in F$. In this paper we show that in many cases the choice of $\lambda \in F$ is completely arbitrary, as long as $\lambda \neq 0$. In Theorem 63 on p. 71 of [1], it is shown that if $n>m$ and $n$ is a prime different from the characteristic of $F$, then $\operatorname{dg}(\alpha+\beta)=m n$. The present result includes this.

THEOREM. Let $E \supseteq F$ be fields as above and let $\alpha, \beta \in E$ with $\operatorname{dg} \alpha=m$, $\operatorname{dg} \beta=n$ and $(m, n)=1$. Then $\operatorname{dg}(\alpha+\lambda \beta)=m n$ for all $\lambda \neq 0, \lambda \in F$ unless the characteristic, $\operatorname{ch}(F)=p$, a prime, and

(a) $p \mid m n$ or $p<\min (m, n)$,

(b) if $m$ or $n$ is a prime power, then $p \mid m n$ and

(c) if $q>m$ for every prime $q \mid n$, then $p \mid n$.

Proof. First we reduce the problem to one of group representations. We may assume without loss that $E$ is a finite degree Galois extension of $F$ and let $G$ be the Galois group. Then $G$ transitively permutes the sets of roots $A=\left\{\alpha_{i} \mid 1 \leqq i \leqq m\right\}$ and $B=\left\{\beta_{j} \mid 1 \leqq j \leqq n\right\}$ of the minimal polynomials of $\alpha$ and $\beta$. Let $V \subseteq E$ be the linear span of $A \cup B$ over $F$. Then $V$ is a $G$-module over $F$ and in the action of $G$ on $V$ there exists orbits $A$ and $B$ with $|A|=m,|B|=n$ and $(m, n)=1$. We show by induction on $|G|$ that if $\alpha \in A$ and $\beta \in B$, then $\alpha+\beta$ lies in an orbit of size $m n$, unless $\operatorname{ch}(F)=p$ and (a), (b) and (c) hold. This will clearly prove the theorem when applied to $\lambda \beta$ in place of $\beta$.

Let $H=G_{\alpha}$ and $K=G_{\beta}$, the stabilizers in $G$ of $\alpha$ and $\beta$. Then $|G: H|=m,|G: K|=n$ and since $(m, n)=1$, a standard argument yields $|G: H \cap K|=m n$ and $H$ and $K$ act transitively on $B$ and $A$ respectively. It follows that $G$ is transitive on $A \times B$ and thus all elements of $V$ of the form $\alpha_{i}+\beta_{j}$ are conjugate under the action of $G$. Suppose that $\alpha+\beta$ does not have exactly $m n$ conjugates. Then not all $\alpha_{i}+\beta_{j}$ are distinct and we may assume that $\alpha+\beta=\alpha_{a}+\beta_{b}$, where

Received by the editors June 6, 1969. 
$\alpha \neq \alpha_{a}$ or $\beta \neq \beta_{b}$. Then $\alpha-\alpha_{a}=\beta_{b}-\beta \neq 0$ and the subspaces $W_{1}$ and $W_{2}$ of $V$, spanned by $A$ and $B$ respectively, intersect nontrivially. Set $U=W_{1} \cap W_{2}$ and observe that $W_{1}, W_{2}$ and $U$ are all $G$-invariant spaces.

We remark at this point that if $\operatorname{ch}(F) \nmid|G|$, an easy contradiction could be obtained using the fact that $W_{1}$ and $W_{2}$ are homomorphic images of the permutation modules determined by the actions of $G$ on $A$ and $B$. In this case, the modules would be completely reducible and since $H K=G$, it is not hard to see that they can have only the principal module as a common constituent. It would follow that $G$ acts trivially on $U$ and thus fixes $\alpha-\alpha_{a}$. A contradiction results since $\alpha_{a}=\alpha^{\sigma}$ for some $g \in G$ and the order of this element is prime to $\operatorname{ch}(F)$. It does not appear that this approach will lead to a full proof of the theorem and we continue along a different route.

It may be assumed that $G$ acts faithfully on $V$ or else the inductive hypothesis may be applied to $G / N$ where $N$ is the kernel of the action, and the result follows immediately. Suppose now that there is a subgroup $G_{0}<G$ which acts so that the orbits $A_{0}$ and $B_{0}$ of $\alpha$ and $\beta$ under $G_{0}$ satisfy $m_{0}\left|m, n_{0}\right| n, \alpha_{a} \in A_{0}$ and $\beta_{b} \in B_{0}$, where $m_{0}=\left|A_{0}\right|$ and $n_{0}=\left|B_{0}\right|$. Then $\left(m_{0}, n_{0}\right)=1$ and since $\alpha+\beta=\alpha_{a}+\beta_{b}$, the number of conjugates of $\alpha+\beta$ under $G_{0}$ is $<m_{0} n_{0}$. Therefore, induction applies and $\operatorname{ch}(F)=p$, a prime, and by (a), $p \mid m_{0} n_{0}$ or $p<\min \left(m_{0}, n_{0}\right)$. Since $m_{0} \mid m$ and $n_{0} \mid n$, (a) holds for $m$ and $n$. Similarly, (b) and (c) for $m_{0}$ and $n_{0}$ imply the corresponding statements for $m$ and $n$. We may assume then that no such subgroup $G_{0}$ exists.

Now, $G$ permutes the set of cosets of $U$ in $W_{1}$ and is transitive on the set of those cosets which contain elements of $A$. All of these, therefore, contain equal numbers of elements of $A$. We have $\alpha$, $\alpha_{a} \in U+\alpha$ and if $A_{0}=A \cap(U+\alpha)$, then $\left|A_{0}\right| \mid m$. Let $G_{0}$ be the stabilizer of the coset $U+\alpha$ in $G$. Clearly, $H \subseteq G_{0}$ and hence $G_{0}$ is transitive on $B$. We claim that $G_{0}$ is transitive on $A_{0}$. If $\alpha_{i} \in A_{0}$, then for some $g \in G, \alpha^{g}=\alpha_{i}$. Thus $(U+\alpha)^{g}=U+\alpha_{i}=U+\alpha$ and so $g \in G_{0}$. This establishes transitivity and by the preceding paragraph, we cannot have $G_{0}<G$. Therefore $G$ stabilizes $U+\alpha$ and hence $A \subseteq U+\alpha$. By similar reasoning, $B \subseteq U+\beta$. Now, $\beta_{j}=u_{j}+\beta$ for some $u_{j} \in U$. Summing over $\beta_{j} \in B$, we obtain $\sum \beta_{j}=\sum u_{j}+n \beta$. Thus $n \beta=u+\gamma$, where $u \in U$ and $\gamma=\sum \beta_{j}$ is fixed by $G$. Let $N \triangleleft G$ be the kernel of the action of $G$ on $A$. Then $N$ fixes all elements of $W_{1} \supseteq U$ and thus $N$ fixes $n \beta$. If $\operatorname{ch}(F) \nmid n$, then $N$ fixes $\beta$ and hence fixes all $\beta_{j}=u_{j}+\beta$. Thus $N$ acts trivially on $V$, the span of $A \cup B$. Therefore, $N=1$ and $G$ is isomorphic to a subgroup of the symmetric group on $A$. Thus $|G| \mid m$ ! and $n \mid m !$. 
Since $n>1$, this shows that the hypotheses of (c) cannot occur if $\operatorname{ch}(F) \nmid n$ and thus (c) is proved.

Now suppose that $\operatorname{ch}(F) \nmid m n$. By interchanging $A$ and $B$ in the above argument, we obtain $|G| \mid n$ ! and all prime divisors of $|G|$ are $\leqq \min (m, n)$. If $\operatorname{ch}(F)=0$ or $\operatorname{ch}(F)=p$, a prime $>\min (m, n)$, then $\operatorname{ch}(F) \nmid|G|$. If $m$ or $n$ is a prime power, we may suppose that $m=q^{e}$ and let $Q$ be a Sylow $q$-subgroup of $K$. Then $|K: K \cap H|=q^{e}$ so $K=(K \cap H) Q$ and it follows that $Q$ is transitive on $A$. Thus under any of the assumptions: $\operatorname{ch}(F)=0, \operatorname{ch}(F)=p>\min (m, n)$ or $m=q^{e}$, there exists a subgroup $L \subseteq K$ which is transitive on $A$ and such that $\operatorname{ch}(F) \nmid|L|$. The proof will be complete if a contradiction follows from the existence of such an $L$.

We have seen that $n \beta=u+\gamma$ where $u \in U$ and $\gamma$ is fixed by $G$. As $U \subseteq W_{1}$, we have $u=\sum \xi_{i} \alpha_{i}$, where $\xi_{i} \in F$ and $\alpha_{i}$ runs over $A$. Now if $x \in L \subseteq K$, we have

$$
\beta=\beta^{x}=\frac{1}{n} \sum \xi_{i} \alpha_{i}^{x}+\frac{1}{n} \gamma .
$$

Now set $\delta=\sum \alpha_{i}$, and observe that since $L$ is transitive on $A$, we have $\sum_{x \in L} \alpha_{i}^{x}=(|L| / m) \delta$. Now, summing $\left(^{*}\right)$ over $L$, we obtain

$$
|L| \beta=\frac{|L|}{m n} \sum \xi_{i} \delta+\frac{|L|}{n} \gamma .
$$

Note that division by $m$ and $n$ in the above equations makes sense in $V$ since $\operatorname{ch}(F) \nmid m n$. Since $\gamma$ and $\delta$ are fixed by $G$ and $\operatorname{ch}(F) \nmid|L|$, it follows that $\beta$ is fixed by $G$. This is a contradiction since $\beta \neq \beta_{b}$ and the proof is complete.

Now let $G$ be any finite group and suppose that $V$ is any faithful finite-dimensional $G$-module over a field $K$. Suppose that $u, v \in V$ are permuted by $G$ into orbits of sizes $m$ and $n$ respectively and that $u+v$ lies in an orbit of size $k$. Then there exist fields $E \supseteq F \supseteq K$, with $E$ a finite separable extension of $F$, and elements $\alpha, \beta \in E$ with $\operatorname{dg} \alpha=m$, $\operatorname{dg} \beta=n$ and $\operatorname{dg}(\alpha+\beta)=k$.

The construction is as follows. Let $e=\operatorname{dim}_{K}(V)$ and let $X_{1}, X_{2}$, $\cdots, X_{e}$ be indeterminates. Set $R=K\left[X_{1}, \cdots, X_{e}\right]$ and let $E$ be the quotient field of $R$. Now fix a basis for $V$ and identify this basis with the $X_{i}$ so that $V$ is identified with the linear span of the $X_{i}$ in $R$. Now it is clear that each element of $G$ determines an automorphism of $R$ and hence of $E$. Let $F$ be the fixed field of $G$ in $E$ and let $\alpha$ and $\beta$ be the elements of $E$ corresponding to $u$ and $v$. These elements clearly have the desired properties. 
It follows that to establish the best possible improvement of the present theorem with conditions given in terms of $m, n$ and $\operatorname{ch}(F)$, it suffices to consider only group representations. It is possible that the theorem could be improved by dropping the possibility $p<\min (m, n)$ in (a). Some limitations on possible improvements are given by the following examples for $m=3$ and $n=4$.

ExAmple 1. $\mathrm{Ch}(K)=2$. Let $G=A_{4}$, the alternating group on four symbols. Let $V^{*}$ be a four dimensional vector space over $G F(2)$ and let $G$ permute a basis, $\{w, x, y, z\}$, in the natural manner. Let $V_{0}=\{0, w+x+y+z\}$ and let $V=V^{*} / V_{0}$. The image of $w$ in $V$ has four conjugates under $G$ and the image of $w+x$ has three conjugates. The sum of these elements has four conjugates.

EXAmple 2. $\mathrm{Ch}(K)=3$. Let $V$ be a four dimensional vector-space over $K=G F(3)$, with basis $\{w, x, y, z\}$. Let $G$ be the group generated by the elements $\rho, \sigma, \tau \in \mathrm{GL}(V)$ whose matrices are

$$
\rho=\left[\begin{array}{llll}
1 & 1 & 0 & 0 \\
0 & 1 & 0 & 0 \\
0 & 0 & 1 & 0 \\
0 & 0 & 0 & 1
\end{array}\right], \quad \sigma=\left[\begin{array}{rrrr}
1 & 0 & 0 & 0 \\
0 & -1 & 0 & 0 \\
0 & 1 & 1 & 0 \\
0 & 1 & 0 & 1
\end{array}\right], \quad \tau=\left[\begin{array}{llll}
1 & 0 & 0 & 0 \\
0 & 1 & 0 & 0 \\
0 & 0 & 0 & 1 \\
0 & 0 & 1 & 0
\end{array}\right] .
$$

Then $G$ is the direct product of the subgroups $\langle\rho, \sigma\rangle$ of order 6 and $\langle\tau\rangle$ of order 2 . The orbit of $w$ under $G$ is $\{w, w+x, w-x\}$ and the orbit of $y$ under $G$ is $\{y, y+x, z, z+x\}$. However, the orbit of $w+y$ is $\{w+y, w+y+x, w+y-x, w+z, w+z+x, w+z-x\}$, which has six elements.

\section{REFERENCE}

1. I. Kaplansky, Fields and rings, Univ. of Chicago Press, Chicago, 1969.

University of Chicago, Chicago, Illinois 60637 